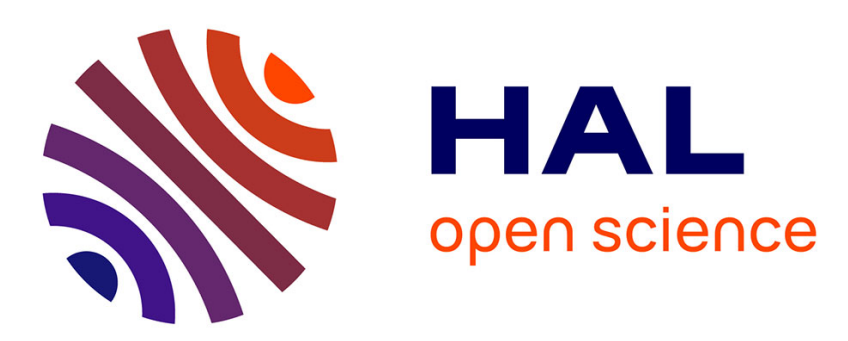

\title{
Critical currents of superconducting aluminium - germanium and lead - germanium thin film alloys near the metal - insulator transition
}

G. Deutscher, M.L. Rappaport

\section{- To cite this version:}

G. Deutscher, M.L. Rappaport. Critical currents of superconducting aluminium - germanium and lead - germanium thin film alloys near the metal - insulator transition. Journal de Physique Lettres, 1979, 40 (10), pp.219-221. 10.1051/jphyslet:019790040010021900 . jpa-00231611

\section{HAL Id: jpa-00231611 \\ https://hal.science/jpa-00231611}

Submitted on 1 Jan 1979

HAL is a multi-disciplinary open access archive for the deposit and dissemination of scientific research documents, whether they are published or not. The documents may come from teaching and research institutions in France or abroad, or from public or private research centers.
L'archive ouverte pluridisciplinaire HAL, est destinée au dépôt et à la diffusion de documents scientifiques de niveau recherche, publiés ou non, émanant des établissements d'enseignement et de recherche français ou étrangers, des laboratoires publics ou privés. 


\title{
Critical currents of superconducting aluminium - germanium and lead - germanium thin film alloys near the metal - insulator transition $\left({ }^{*}\right)$
}

\author{
G. Deutscher and M. L. Rappaport \\ Department of Physics and Astronomy, Tel-Aviv University, Tel-Aviv, Israel
}

(Reçu le 27 février 1979, accepté le 28 mars 1979)

\begin{abstract}
Résumé. - Les courants critiques de films minces d'alliages $\mathrm{Al}-\mathrm{Ge}$ et $\mathrm{Pb}-\mathrm{Ge}$ ont été mesurés en fonction de la concentration en volume de métal $x$. Nous avons trouvé qu'ils varient comme $\left(x-x_{\mathrm{c}}\right)^{v}$ où $x_{\mathrm{c}}$ est la valeur de $x$ à la transition métal-isolant. Ce comportement est similaire à celui de la conductance $\sigma$ des mêmes échantillons à l'état normal. Nous interprétons ces résultats dans le cadre des théories récentes du phénomène de percolation.

Abstract. - The critical currents of $\mathrm{Al}-\mathrm{Ge}$ and $\mathrm{Pb}-\mathrm{Ge}$ thin film alloys have been measured as a function of the metal volume fraction $x$. They have been found to vary as $\left(x-x_{\mathrm{c}}\right)^{v}$ where $x_{\mathrm{c}}$ is the value of $x$ at the metal-insulator transition. This behaviour is similar to that of the conductances $\sigma$ of the same samples in the normal state. We interpret these results in terms of current percolation theories.
\end{abstract}

We report in this letter the first critical current measurements on superconductor-semiconductor mixtures as a function of the metal volume fraction $x$ near the metal-insulator threshold $x_{\mathrm{c}}$, and we propose an interpretation of the results in terms of the percolation model of de Gennes [1] and Skal and Shklovskii [2]. This interpretation leads to the conclusion that the critical current $j_{\mathrm{c}} \propto\left(x-x_{\mathrm{c}}\right)^{v}$ with $v=(d-1) v$ where $d$ is the dimensionality of the sample and $v$ the critical index for the coherence length relevant to the percolation problem. In the model of references [1] and [2] the critical index $t$ for the normal state conductance $\sigma$ is given by $t=(d-1) v+\delta$ where $\delta$ is a twistedness index for the percolative paths $(\delta>0)$. Experimentally we find within our experimental uncertainty that $v=t=1.75 \pm 0.15$ in 3D Al-Ge films and we conclude that the twistedness of the percolative paths does not actually decrease the conductance of the network, which is in practice governed (like the critical current density in the superconducting state) only by the density of percolative paths. In the (probably) 2D Pb-Ge films,

$$
v=1.3 \pm 0.1 \text { and } t=0.9 \pm 0.1:
$$

$\left(^{*}\right)$ Research supported by the Israel National Council for Research and Development and the Karlsruhe Nuclear Research Centre. it appears that in this regime the model of Skal and Shklovskii is not valid since $\delta<0$.

The electrical properties of metal-insulator mixtures have recently been the subject of considerable attention [3], [4]. Measurements have primarily concentrated on the rapid change of $\sigma$ near the metalinsulator transition. In accordance with percolation theory [5] it has been observed that $\sigma \propto\left(x-x_{\mathrm{c}}\right)^{t}$ where $t$ falls in general in the range from 1.5 to 1.9 , both experimentally and from theoretical estimates. However, the exponent $t$ is not directly related to other exponents of the percolation problem for which renormalization group theories make accurate predictions. Moreover, experimental determinations of $t$ suffer from the fact that $\sigma$ does not actually go to zero at $x=x_{\mathrm{c}}$ due to the finite conductivity of the matrix and to quantum effects [6].

The advantages of critical current measurements are that they are related to the better known exponent $v$ and that at concentrations $x<x_{c}$ the critical current is zero. We have performed measurements on $\mathrm{Pb}-\mathrm{Ge}$ $\left(x_{\mathrm{c}}=18 \%\right)$ and $\mathrm{Al}-\mathrm{Ge}\left(x_{\mathrm{c}}=55 \%\right)$ thin film alloys, where the former system has a random structure and a value of $x_{c}$ in accordance with that predicted by percolation theory in a random continuous medium while the latter has a highly regular granular structure [7] and a value of $x_{c}$ close to that of a close packed array of spheres. 
Samples were prepared by evaporation of the constituents from two electron beam guns onto room temperature glass substrates at a pressure of $5 \times 10^{-6}$ torr during evaporation. The evaporation rate from each gun was monitored and controlled through a separate quartz thickness monitor. Evaporation rates were held constant to whithin a few percent. Nine samples of thickness close to $2000 \AA$ were deposited simultaneously on each substrate, sample composition varying across the substrate due to varying distances from the evaporation sources. Sample concentrations were established using the quartz thickness monitor readings and deposition profiles measured for each material by interferometry. Concentration difference between neighbouring samples $(8.5 \mathrm{~mm}$ apart) was from one to two percent, depending on the particular concentration under investigation. This set up allows a fairly good knowledge of the concentration differences between samples deposited on the same substrate, while the absolute value of the concentration is only known to within several percent.

Normal state conductivities $\sigma$, critical temperatures $T_{\mathrm{c}}$, and critical current densities $j_{\mathrm{c}}$ were measured on the same samples by the standard four point contact method. Critical current densities were determined as that producing a $1 \mu \mathrm{V}$ potential difference across the sample (1 mm in length). In order to study concentration dependences near the metal insulator transition we used samples evaporated on a single substrate with a median concentration slightly above the threshold $x_{\mathrm{c}}$. The value of $x_{\mathrm{c}}$ was chosen as that for which $j_{\mathrm{c}}$ went to zero. For Pb-Ge $\left(T_{\mathrm{c}} \simeq 7.2 \mathrm{~K}\right) j_{\mathrm{c}}$ was measured at $4.2 \mathrm{~K}$ and for $\mathrm{Al}-\mathrm{Ge}\left(T_{\mathrm{c}} \simeq 1.8 \mathrm{~K}\right)$ at $1.42 \mathrm{~K}$.

The results obtained for Al-Ge are presented in the form of a log-log plot in figure 1 , which shows that both $\sigma$ and $j_{\mathrm{c}}$ vary as $\left(x-x_{\mathrm{c}}\right)^{1.75 \pm 0.15}$. The value obtained for the exponent depends somewhat upon the exact choice made for $x_{\mathrm{c}}$, but this does not affect the main conclusion that the behaviour of $j_{\mathrm{c}}$ and $\sigma$ near $x_{c}$ are essentially the same. The value of 1.75 is in good agreement with that predicted for $t$ by percolation theory for a 3D medium [8] (actually the Al-Ge films contain about 20 layers of $\mathrm{Al}$ grains so that the crossover from 3D to $2 \mathrm{D}$ would occur only very close to $x_{\mathrm{c}}$ ). The departure from linearity observed for $\log \sigma$ at low values of $\log \left(x-x_{c}\right)$ may be due to the finite conduction of the Ge matrix and/or to quantum effects [6]. In $\mathrm{Pb}-\mathrm{Ge}$ (Fig. 2) the critical index $v$ is somewhat smaller than in Al-Ge $(1.3 \pm 0.1)$, possibly due to the fact that the film thickness in only about 4 times the grain size so that the films are 2D rather than $3 \mathrm{D}$ in the investigated range $x-x_{\mathrm{c}}<0.1$. Moreover, it is seen in figure 2 that $t<v$. The slope of the conductance data $(0.9 \pm 0.1)$ may be misleading due to the contribution of the Ge near $x_{c}$, but is consistent with the calculated value of 1.1 in $2 \mathrm{D}$ [8].

We now turn our attention to the comparison between the behaviour of $j_{\mathrm{c}}$ and that of $\sigma$, which we

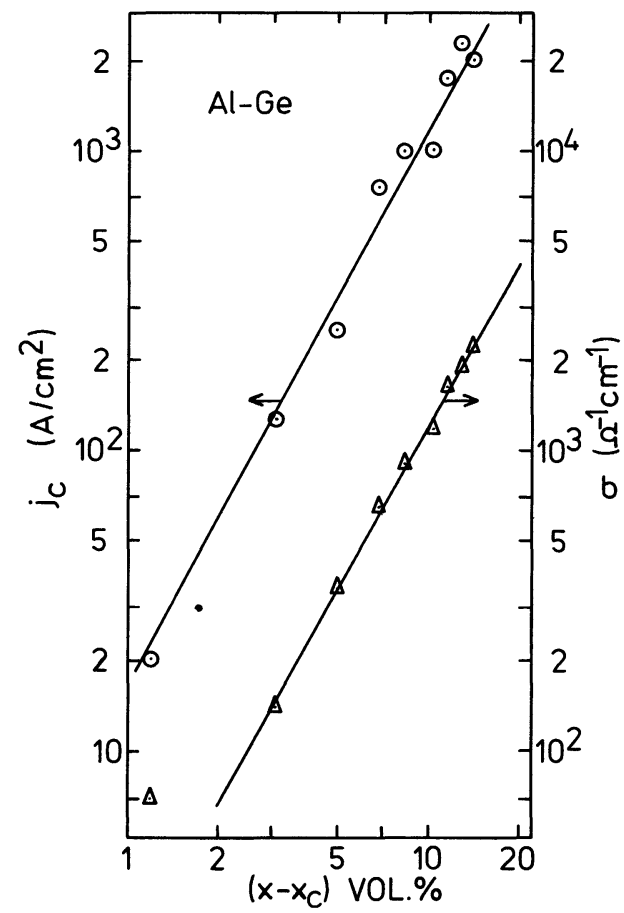

Fig. 1. - Log-log plot of the critical current density $j_{\mathrm{c}}$ and normal state conductance $\sigma$ versus concentration in 3D Al-Ge, showing straight lines with slopes equal to $1.75 \pm 0.15$.

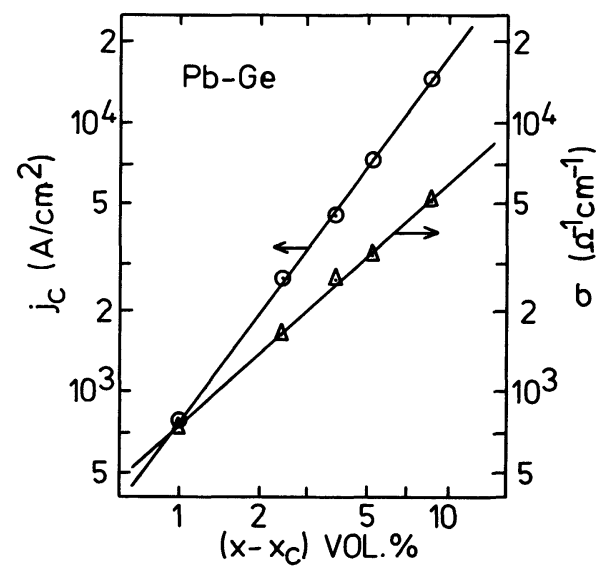

Fig. 2. - Log-log plot of the critical density $j_{\mathrm{c}} \propto\left(x-x_{\mathrm{c}}\right)^{v}$ and normal state conductance $\sigma \propto\left(x-x_{\mathrm{c}}\right)^{t}$ in $2 \mathrm{D} \mathrm{Pb}=\mathrm{Ge}$ showing that $v(=1.3 \pm 0.1)>t(=0.9 \pm 0.1)$.

propose to interpret with the percolation model of de Gennes [1] and Skal and Shklovskii [2]. In this model, the current flows along macrobounds. The average distance between nodes is seen as the correlation length $\xi$ of the percolation problem and varies as $\left(x-x_{c}\right)^{-v}$ while the average length of a macrobond between neighbouring modes $L$ varies as $\left(x-x_{\mathrm{c}}\right)^{-v-\delta}$ where $\delta>0$. The conductivity varies as $L^{-1} \xi^{2-d}$ and therefore its critical index

$$
t=(d-1) v+\delta
$$

depends both on the density of the macrobond network and on the twistedness index $\delta$. 
The critical current is defined experimentally by the appearance of a certain flux flow voltage across the sample. The flux flow voltage that appears between two nodes due to flux creep through the intergrain junctions can be written as

$$
V \propto N r I \exp -\frac{E_{\mathrm{c}}-\frac{\hbar}{2 e} I}{k T}
$$

where $N$ is the number of intergrain junctions along the macrobond, $r$ the normal state resistance of one junction and $E_{\mathrm{c}}$ its Josephson coupling energy. At low temperatures $k T<E_{\mathrm{c}}$ the critical current $I_{\mathrm{c}}$ (the value of the current at which a certain flux flow voltage $V_{0}$ appears) is essentially determined by $E_{\mathrm{c}}$ and depends only logarithmically on $V_{0}$ and the macrobond length. In this regime the critical current density of the sample is $j_{\mathrm{c}}=I_{\mathrm{c}} \xi^{1-d}$ so that $v=(d-1) v$.

Therefore a comparison between the values of $t$ and $v$ measured on the same set of samples allows a determination of the twistedness index $\delta$. Our measurements on the 3D Al-Ge alloys give

$$
t=2 v=1.75 \pm 0.15
$$

in excellent agreement with the contention of Skal and Shklovskii [2] that $\delta \ll 1$ and with numerical estimates of $t$ and $v$ [8]. The critical current data obtained for $\mathrm{Pb}-\mathrm{Ge}-$ if we interpret them as characteristic of a
2D layer due to the rather small thickness to grain size ratio $(\sim 4)$ - gives $v=v=1.3 \pm 0.1$, again in excellent agreement with calculated values [8]. The conductance data give - with the reservation mentioned above $-\mathrm{a}$ value of $t=0.9 \pm 0.1$ which is smaller than $v$. Such a result seems to invalidate the Skal and Shklovskii picture for $d=2$. For that case we note that calculated values of $t$ are actually smaller than $v[8]$.

In summary, by a comparison of conductance and critical current measurements on the same set of samples we have established experimentally for the first time that the twistedness of the percolation paths does not play an important role in the conductance of the network, as first suggested by Skal and Shklovskii. From the critical current data we have obtained values for the critical index $v$ in $3 \mathrm{D}(0.87 \pm 0.08)$ and in $2 \mathrm{D}(1.3 \pm 0.1)$ that are in good agreement with calculated values [8]. And finally, in 2D the slower variation of the conductance as compared to the critical current (corresponding to a negative value of the twistedness index $\delta$ ) seems to invalidate the model of Skal and Shklovskii for that dimensionality, a point already mentioned by Kirkpatrick [8] on the basis of computer simulations.

Acknowledgments. - It is a pleasure to thank P. G. de Gennes for pointing out to us reference [2] and for a most valuable discussion of the critical current data and their interpretation.

References

[1] De Gennes, P. G., J. Physique Lett. 37 (1976) L-1.

[2] SKAL, A. S. and ShKLovsKII, B. I., Fiz. Tekh. Poluprodn. 8 (1974) 1586 ; [Sov. Phys. Semicond. 8(1975) 1029].

[3] ABeles, B., in Applied Solid State Science, edited by R. Wolfe (Academic Press, New York) 1976, Vol. 6, p. 1 and Adv. Phys. 24 (1975) 407.

[4] CoutTs, T. J., Thin Solid Films 38 (1976) 313.

[5] KIRKPATRICK, S., lecture presented at the Les Houches Summer School in Physics, 1978, to be published by North Holland.
[6] Friedel, J., J. Physique Lett. 37 (1976) L-9.

[7] DeutsCher, G., RaPPAPORT, M. and OVADYAhu, Z., Solid State Commun. 28 (1978) 593.

[8] Generally accepted calculated values of $v$ are $1.2-1.3$ (2D), $0.8-0.9$ (3D) and values of $t$ are 1.1 (2D), $1.6-1.7$ (3D). Values quoted by KirkPatrick, S., AIP Conf. Proc. 40 (1978) 99 and STRAley, J. P., ibid., p. 118 